\title{
To predict the radiosensitivity of nasopharyngeal carcinoma using intravoxel incoherent motion MRI at $3.0 \mathrm{~T}$
}

\author{
Wen Bo Chen2, Bin Zhang1,3, Long Liang1, Yu Hao Dong ${ }^{1,4}$, Guan Hui Cai², Chang \\ Hong Liang ${ }^{1}$, Bo Wen Lan ${ }^{2}$ and Shui Xing Zhang ${ }^{1}$ \\ ${ }^{1}$ Department of Radiology, Guangdong Academy of Medical Sciences/Guangdong General Hospital, Guangzhou, Guangdong, \\ P.R. China \\ ${ }^{2}$ Department of Radiology, HuiZhou Municipal Central Hospital, Huizhou, Guangdong, P.R. China \\ ${ }^{3}$ Southern Medical University, Guangzhou, Guangdong, P.R. China \\ ${ }^{4}$ Shantou University Medical College, Shantou, Guangdong, P.R. China \\ Correspondence to: Shui Xing Zhang, email: shui7515@126.com
}

Bo Wen Lan, email: chlliu2@163.com

Keywords: intravoxel incoherent motion, MRI, nasopharyngeal carcinoma, IMRT, radiosensitivity

Received: August 04, $2016 \quad$ Accepted: April 11,2017 Published: April 21, 2017

Copyright: Chen et al. This is an open-access article distributed under the terms of the Creative Commons Attribution License 3.0 (CC BY

3.0), which permits unrestricted use, distribution, and reproduction in any medium, provided the original author and source are credited.

\section{ABSTRACT}

Purpose: To investigate intravoxel incoherent motion (IVIM) MRI for evaluating the sensitivity of radiotherapy on nasopharyngeal carcinoma (NPC).

Results: The reproducibility between intra-observer and inter-observer was relatively good. $D\left(0.72 \times 10^{-3} \mathrm{~mm}^{2} / \mathrm{s} \pm 0.14\right.$ vs. $\left.0.54 \times 10^{-3} \mathrm{~mm}^{2} / \mathrm{s} \pm 0.23 ; P<0.001\right)$ and $D *\left(157.92 \times 10^{-3} \mathrm{~mm}^{2} / \mathrm{s} \pm 15.21\right.$ vs. $\left.120.36 \times 10^{-3} \mathrm{~mm}^{2} / \mathrm{s} \pm 10.22 ; P<0.0001\right)$ were significantly higher in effective group than poor-effective group, whereas the difference of $f(18.79 \% \pm 2.51$ vs. $16.47 \% \pm 1.51)$ and $A D C\left(1.21 \times 10^{-3} \mathrm{~mm}^{2} / \mathrm{s} \pm 0.11\right.$ vs. $1.33 \times 10^{-3} \mathrm{~mm}^{2} / \mathrm{s} \pm 0.23$ ) could not reach statistical significant between the 2 groups $(P>0.05)$.

Conclusions: IVIM may be potentially useful in assessing the radiosensitivity of NPC. The higher $D$ value combining with higher $D *$ value might indicate the more radiosensitive of NPC, and increased $D *$ might reflect increased blood vessel generation and parenchymal perfusion in NPC.

Materials and Methods: Sixty consecutive patients (20 female, range, 27-83 years, mean age, 52 years) newly diagnosed NPC in the stage of T3 or T4 were enrolled. Forty-two of them were divided into effective group clinically after a standard radiotherapy according to the RECIST criteria. IVIM with 13 b-values (range, 0-800 s/ $\mathrm{mm}^{2}$ ) and general MRI were performed at 3.0T MR scanner before and after radiotherapy. The parameters of IVIM including perfusion fraction $(f)$, perfusionrelated diffusion $\left(D^{*}\right)$, pure molecular diffusion $(D)$ and apparent diffusion coefficient $(A D C)$ were calculated. Two radiologists major in MRI diagnose analyzed all images independently and placed regions of interest (ROIs). Intra-class correlation coefficient (ICC) was used to evaluate intra-observer and inter-observer agreement. And MannWhitney test was used to assess the differences between the two groups.

\section{INTRODUCTION}

Nasopharyngeal carcinoma (NPC) is a geographic and racial malignancy in global nation, especially in southern China with a high incidence up to $15-24 / 100,000$ [1]. Nowadays, radiotherapy plays a very important role in the treatment of NPC, and intensity-modulated radiotherapy (IMRT) is regarded as a major breakthrough. However, a long-term outcome of large NPC series suggested up to $20 \%$ patients failed because of distant metastasis (DM) after IMRT [2]. For the failed patients, a taxane-based chemotherapy would be used to reduce DM. 
Therefore, it is obvious that a non-invasive and accurate technique for evaluating the radiosensitivity of NPC is urgent and can help determine whether chemotherapy or immunotherapy combination is needed or not.

Due to its superior soft tissue contrast resolution, general MRI was mainly used for morphological diagnosis and staging of NPC. Unfortunately, with the defect in assessing the tumor microenvironment, both general MRI and CT are limited in evaluating the radiosensitivity of NPC. The microenvironment of tumor including interstitial hypertension and hypoxia is associated with DM and treatment failure [3, 4]. While emerging clinical data suggests that alleviating tumor hypoxia by improving tumor perfusion or oxygenation may actually enhance the outcome of radiotherapy, chemotherapy, and immunotherapy [5]. And oxygenation depends on both $\mathrm{O} 2$ diffusion and blood perfusion. Therefore, the tumor microenvironment including diffusion and perfusion can probably reveal the radiosensitivity of NPC.

Diffusion-weighted (DW) MR imaging enhances contrast ratio in the tissues based on the different water diffusion in distinct tissues. Besides, DWI could be used to differentiate the histological types in certain malignancies [6-8]. However, the effect of diffusion in tissues can substantially be confounded by perfusion because of the Brownian motion of $\mathrm{H}+$ in pseudorandom capillary networks [9]. IVIM, a special DWI technique with several b values, can separate quantificational effects of diffusion and perfusion $[10,11]$. Many studies revealed that IVIM was advantageous in demonstrating superior results in head and neck cancers compared with general DWI [1214]. Therefore, IVIM was much more advantageous and useful for evaluating the radiosensitivity of NPC than general DWI.

According to IVIM theory [9], MR signal attenuation could be expressed using four parameters $\left(f, D, D^{*}, A D C\right)$ in a biexponential equation [15]. Pilot studies explored the value of IVIM in head and neck cancers $[14,16]$. And in our prior study [17], we had found $D(P=0.001)$ and $f$ $(P<0.0001)$ were significantly lower in patients with NPC than enlarged adenoids, whereas $D^{*}$ was significantly higher $(P<0.0001)$, and increased $D^{*}$ indicated blood vessel generation and increased parenchymal perfusion in primary NPC. Although we had established a system using IVIM DWI to differentiate the tumors in nasopharyngeal region, while a very few published studies had explored IVIM in evaluating the radiosensitivity of NPC. Thus, the aim of this research is to obtain the $D, D^{*}, A D C$ and $f$ values using IVIM theory and explore the value of IVIM in evaluating the radiosensitivity of NPC.

\section{RESULTS}

General MRI and IVIM were successfully performed in the 60 patients using 3.0-T whole-body system (Signa EXCITE HD, GE Healthcare, Milwaukee, WI, USA) before and after standard regimen of IMRT. The images of general MR sequence were showed in Figure

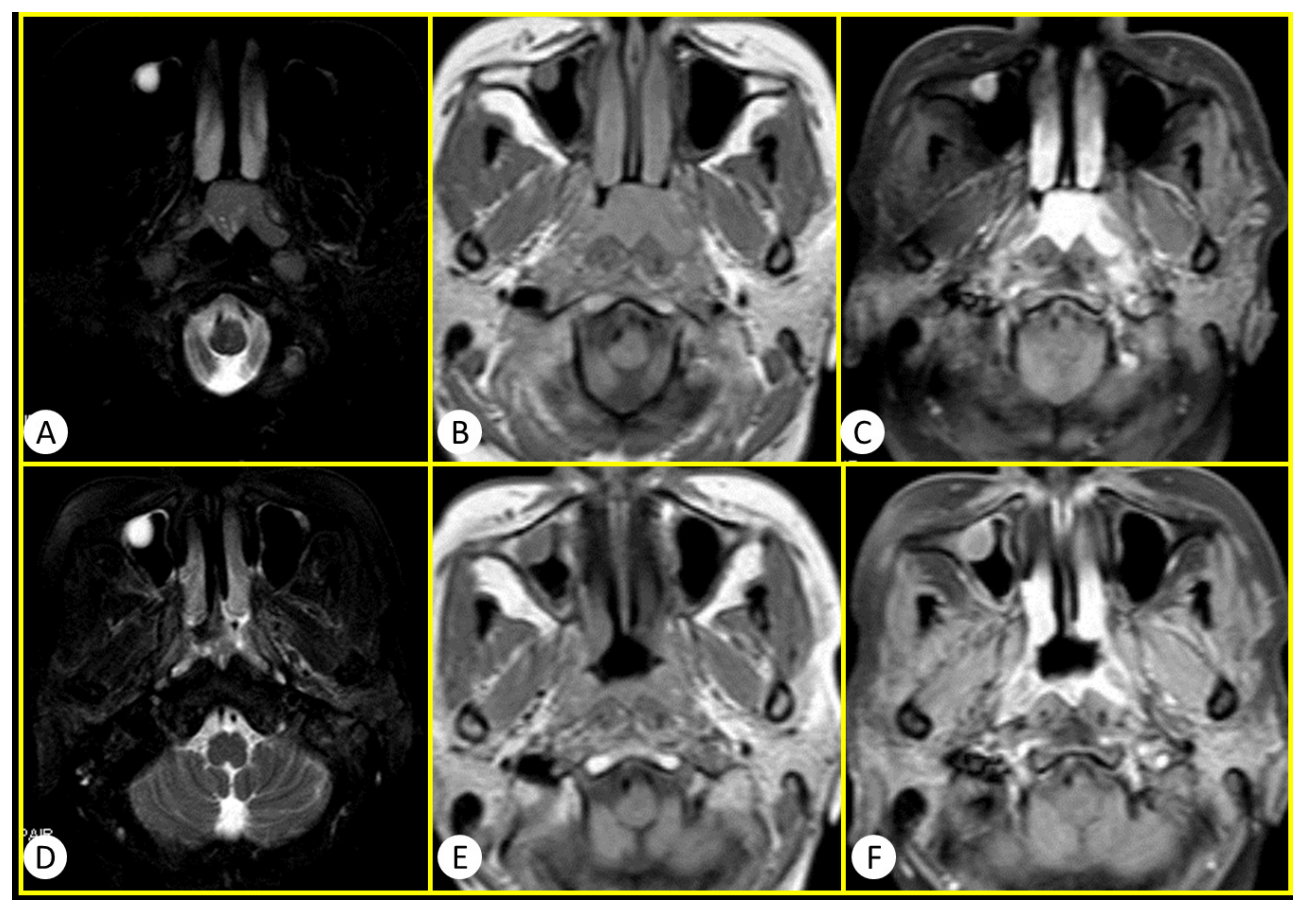

Figure 1: The conventional MR images of a 50 years old woman diagnosed as NPC. A.-C. were before the therapy of IMRT and D.-F. were after the therapy of IMRT. A and D were $\mathrm{T}_{2}$ WI SPAIR. B and D were $\mathrm{T}_{1}$ WI. C and F were $\mathrm{T}_{1}$ WI enhanced. Compared with the images before IMRT, the longest diameter of tumor lesion was obviously diminish $>50 \%$ after the therapy of IMRT. Therefore this case was sort into the effective group with PR according to the RECIST standard. 


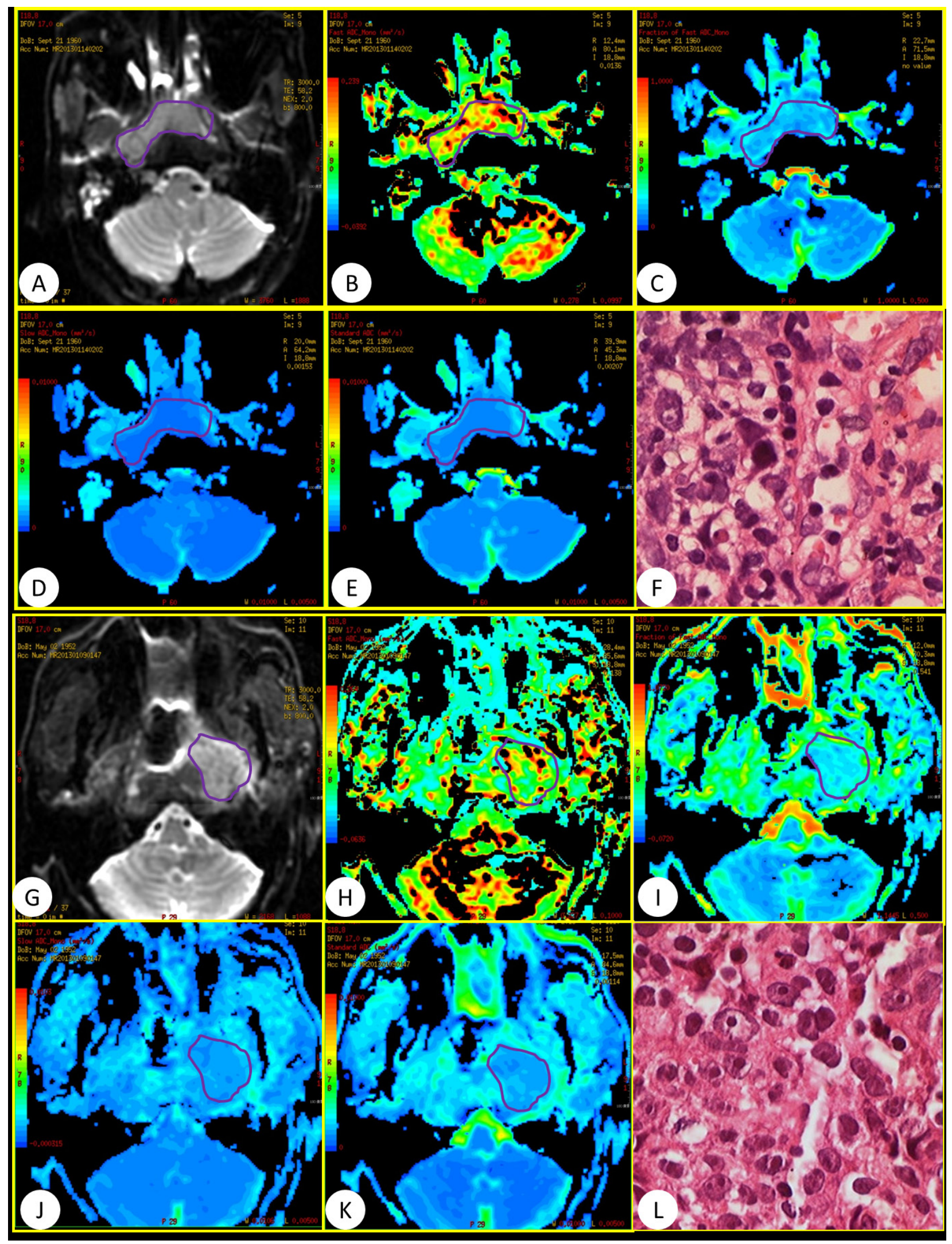

Figure 2: (A-F) Was a 52-year-old man in effective group and $(\mathbf{G}-\mathbf{L})$ was a 60 -year-old women in poor effective group who referred to our department for confirmation of the NPC diagnosis. IVIM with $13 \mathrm{~b}$ values (in the range $0-800 \mathrm{~s} / \mathrm{mm} 2$ ) was performed before standard regimen of IMRT. (A and G) DWI image, (B and H) D*, (C and I). $\mathrm{f}$, (D and J) D, (E and K): ADC parameters, (F and L) pathological slides. 
Table 1: The values of the IVIM parameters for NPC of the two groups before IMRT

\begin{tabular}{lcccc}
\hline & $\mathbf{D}\left(\times \mathbf{1 0} \mathbf{1 0}^{-3} \mathbf{m m}^{2} / \mathbf{s}\right)$ & $\mathbf{D} *\left(\times \mathbf{1 0} \mathbf{0}^{-3} \mathbf{m m}^{2} / \mathbf{s}\right)$ & $\mathbf{f}(\mathbf{\%})$ & $\mathbf{A D C}\left(\times \mathbf{1 0} \mathbf{1}^{-3} \mathbf{m m}^{\mathbf{2}} / \mathbf{s}\right)$ \\
\hline Effective group & $0.72 \pm 0.14$ & $157.92 \pm 15.21$ & $18.79 \pm 2.51$ & $1.21 \pm 0.11$ \\
$(42 / 60)$ & & & \\
Poor group & $0.54 \pm 0.23$ & $120.36 \pm 10.22$ & $16.47 \pm 1.51$ & $1.33 \pm 0.23$ \\
$(18 / 60)$ & & & \\
P value & $<0.001^{*}$ & $<0.0001^{*}$ & $>0.05$ & $>0.05$ \\
\hline
\end{tabular}

Note: Unless otherwise indicated, data are means \pm standard deviations.

*Mann-Whitney test for differences in IVIM parameters between the two groups.

1. IVIM images and representative pathological slides of biopsy for both effective groups and poor effective groups were showed in Figure 2. The mean largest diameters of tumor $( \pm \mathrm{SD})$ in patients with NPC before and after IMRT were showed in Figure 3, respectively.

There were excellent inter-observer agreements in IVIM parameters $D$ with ICC value of 0.937 (95\% CI: $0.926-0.968)$ and $f$ with ICC value of 0.922 (95\% CI: $0.920-0.962)$, and a relatively good ICC value of $D^{*}$ with $0.902(95 \%$ CI: $0.851-0.909)$. The result suggested excellent agreement between the 2 readers for all IVIM measures.

The values of the IVIM parameters for NPC of the two groups before IMRT were showed in Table 1. The classification results obtained from Leave-one out tests of original grouped cases and cross-validated grouped cases were $95.0 \%$ and $93.3 \%$.

Box plots comparing $D, D^{*}, f$ and $A D C$ between the two groups are shown in Figure 4. As shown in Figure 5, the ROC curves indicated that when both sensitivity and

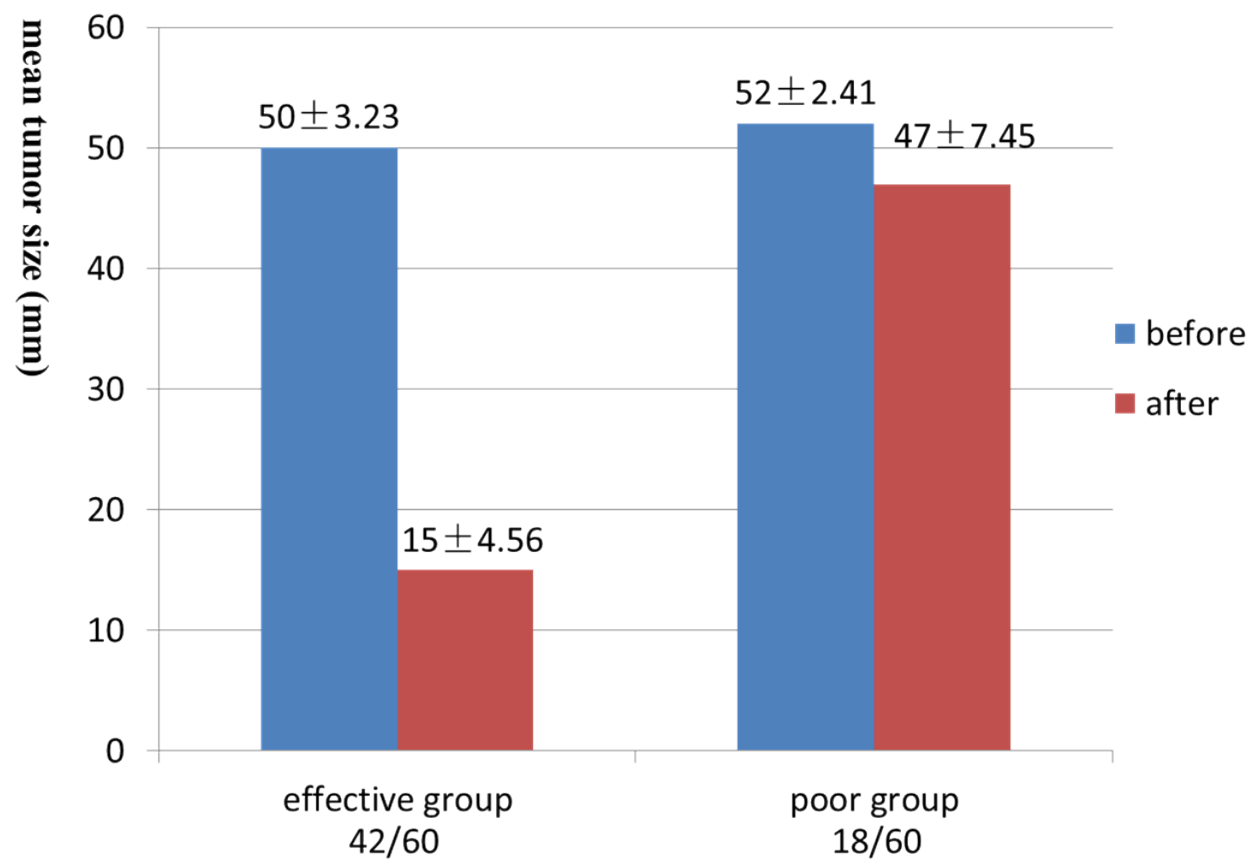

Figure 3: The mean tumor size before and after IMRT. The mean largest diameter of lesions in effective group after IMRT was significantly decrease compared with before $(50 \pm 3.23 \mathrm{~mm}$ vs. $15 \pm 4.56 \mathrm{~mm}, P<0.05)$. And the difference in the poor group could not reach statistical significance $(52 \pm 2.41 \mathrm{~mm}$ vs. $47 \pm 7.45 \mathrm{~mm}, P>0.05)$. 
specificity were adjusted to produce the highest Youden index, the optimal $D$ and $D^{*}$ threshold for distinguishing radiosensitive NPC were $0.593 \times 10^{-3} \mathrm{~mm}^{2} / \mathrm{s}$ and $147.5 \times 10^{-}$ ${ }^{3} \mathrm{~mm}^{2} / \mathrm{s}$ respectively. Besides the AUC for $D^{*}(0.942)$ was a little larger than $D(0.941)$. The sensitivity of $D$ and $D^{*}$ were $93.33 \%$ and $97.37 \%$. The specificity of $D$ and $D^{*}$ were $60 \%$ and $50 \%$. Histogram analysis (Figure 6 ) demonstrated that all the values of $D, D^{*}$ and $f$ were relatively stable and acceptable, while $A D C$ values of 5/18 in poor effective group were quirky and illogic high.

\section{DISCUSSION}

Comparing to the general DWI model, the IVIM fits the signal decay with a biexponential decay and the parameters may reflect water diffusion and blood perfusion more accurately [9]. By this model, the parameters (D, $\left.f, D^{*}, A D C\right)$ are estimated simultaneously; $f$ and $D^{*}$ are related to blood perfusion, and $D$ and $A D C$ are related to water diffusion [18-20]. The IVIM model was recently applied to investigate human placental function, renal perfusion, characterize prostate and breast tumors [2124]. However, we applied the IVIM model to evaluate the radiosensitivity of NPC. In our study, local shim, parallel imaging, and a reduced echo time were used to insure high quality IVIM imaging, which was confirmed to be useful in our prior research [17].

Using an 11-b DW imaging sequence, Guiu et al. [25] reported that seven b values $(0,5,15,25,35,50$ and $100 \mathrm{~s} / \mathrm{mm}^{2}$ ) could model the first portion of the biexponential decay curve. The number of $b$-values used for extracting perfusion-sensitive information during DWI varies between studies and ranges from four to more than ten. A larger number of b-values provided more powerful
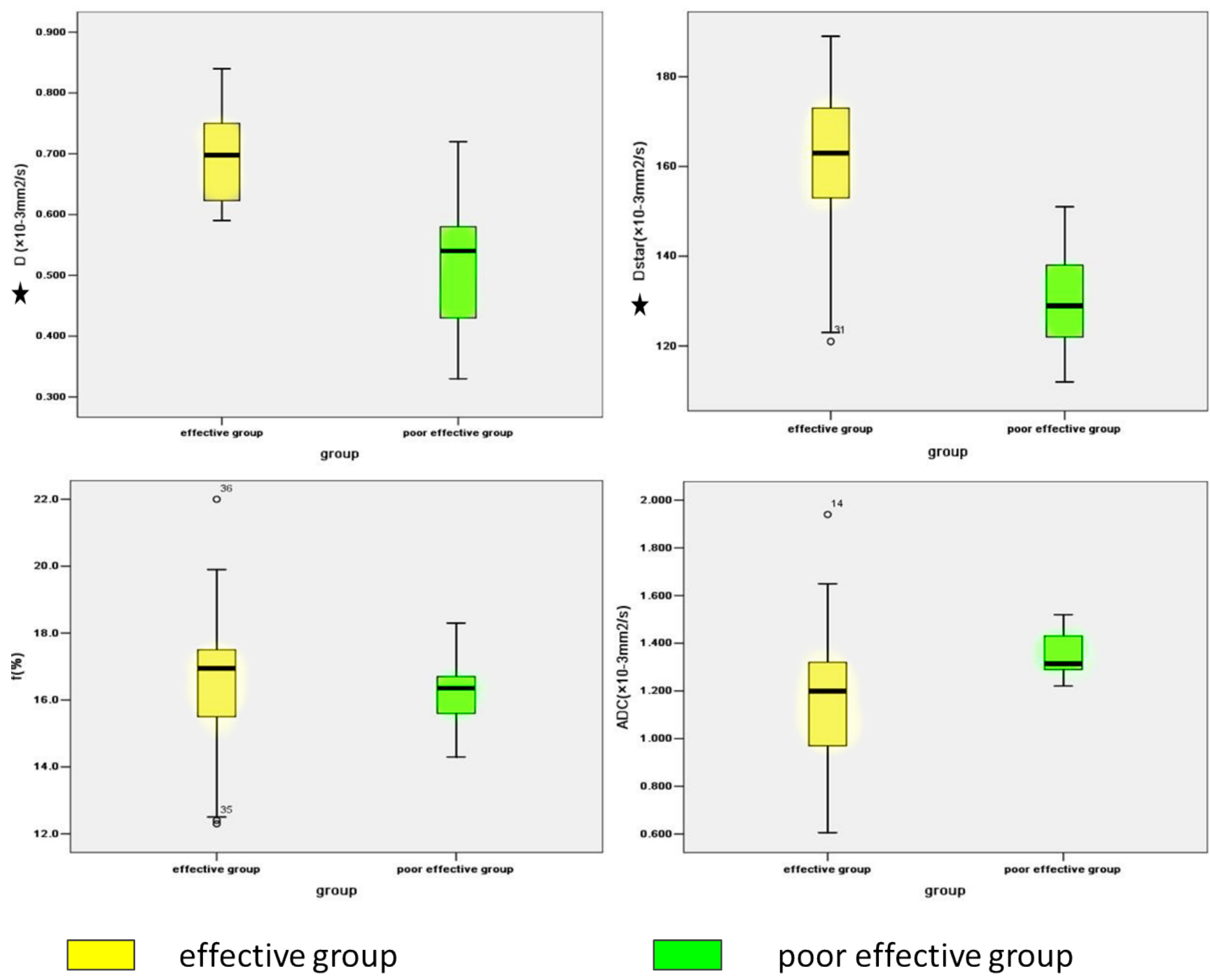

Figure 4: Box plots comparing $\boldsymbol{D}, \boldsymbol{D}^{*}, \boldsymbol{f}$ and $\mathbf{A} \boldsymbol{D} \boldsymbol{C}$ between the two groups. From the box plots, it is obvious that $D$ and $D^{*}$ were significantly higher in effective group than poor-effective group, whereas the difference of $f$ and $A D C$ could not reach statistical significant. *means $P<0.05$. 
data supporting the estimate and, in particular, enabling the uncertain parameter to be measurable. The accuracy of estimating $D^{*}$ improved with sampling (i.e., as the number of b-values increased) in the $0-200 \mathrm{~s} / \mathrm{mm}^{2}$ range [26, 27]. Susceptibility artifacts increase with the use of higher b-values, typically around $1000 \mathrm{~s} / \mathrm{mm}^{2}$. Furthermore, the poor signal-to noise ratio (SNR) obtained at higher b-values decreases the accuracy of IVIM calculations. Consequently, we tried to minimize this effect by setting the maximum b-value to be $800 \mathrm{~s} / \mathrm{mm}^{2}$. In our study, $13-\mathrm{b}$ values were used, and nine of these were applied to model this region of the bi-exponential curve. As a result, the reproducibility of the IVIM parameters both in intraobserver and inter-observer was relatively good .

In our study, we found that $D$ value was significantly higher in effective group than in poor-effect group $\left(0.72 \times 10^{-3} \quad \mathrm{~mm}^{2} / \mathrm{s} \pm 0.14\right.$ vs. $0.54 \times 10^{-3} \mathrm{~mm}^{2} / \mathrm{s} \pm 0.23$; $P<0.001$ ), indicating that $D$ was restricted in the NPC cases with poor radiosensitivity. Our finding was similar with other studies indicated cancers present a greater impediment to diffusion due to the more densely packed tumor cells and more cell membranes. In other words, the decrease in the number of cells or necrosis during treatment leaded to smaller impediment to diffusion [28, 29]. Simultaneously, the data suggested that IVIM had greater clinical potential to assess the radiosensitivity of tumors based on their $D$ values with a threshold of $0.593 \times 10^{-3} \mathrm{~mm}^{2} / \mathrm{s}$. The significant differences of $D$ values between the two groups of NPC may due to the differences of cellularity and extracellular matrix composition. In the present preclinical study [30], tumors subjected to DWMRI were examined with respect to cell density, fraction of hypoxic tissue, level of interstitial hypertension, and metastatic status. The low $D$ values were found to have high cell densities, indicating restricted Brownian motion of water [31-33]. The increased cellularity and varying amounts of stromal tissues would reduce molecular diffusivity [34], the same as oxygen diffusion and cause hypoxia which resulted in the poor radiosensitivity of IMRT in NPC. Therefore, $D$ might be useful in clinical practice to help evaluate the radiosensitivity of NPC before IMRT. On the other hand, despite the statistically significant differences in the overall $D$ between the two groups, the D values vary considerably and, importantly, overlapped appreciably between the two groups of cases.

Normally, $D$ and $A D C$ showed the same patterns that higher $D$ value should associated with higher $A D C$ value in effective group. When b value $>200 \mathrm{~s} / \mathrm{mm}^{2}$, the $A D C$ value simply corresponds to $D$, which is the true water diffusion coefficient including both intra- and extracellular water diffusion. Using $4 \mathrm{~b}$ values $(200,400,600,800 \mathrm{~s} /$ $\mathrm{mm}^{2}$ ) to obtain a more accurate $A D C$ value, we aimed at exploring whether the difference of ADC values between the two groups was statistical significant or not. But we found that the value of $\mathrm{ADC}\left(1.21 \times 10^{-3} \mathrm{~mm}^{2} / \mathrm{s} \pm 0.11\right.$ vs. $1.33 \times 10^{-3} \mathrm{~mm}^{2} / \mathrm{s} \pm 0.23$ ) could not reach statistical significance. While in our study, $5 / 18$ in poor effective group got quirky high value which made the mean $A D C$
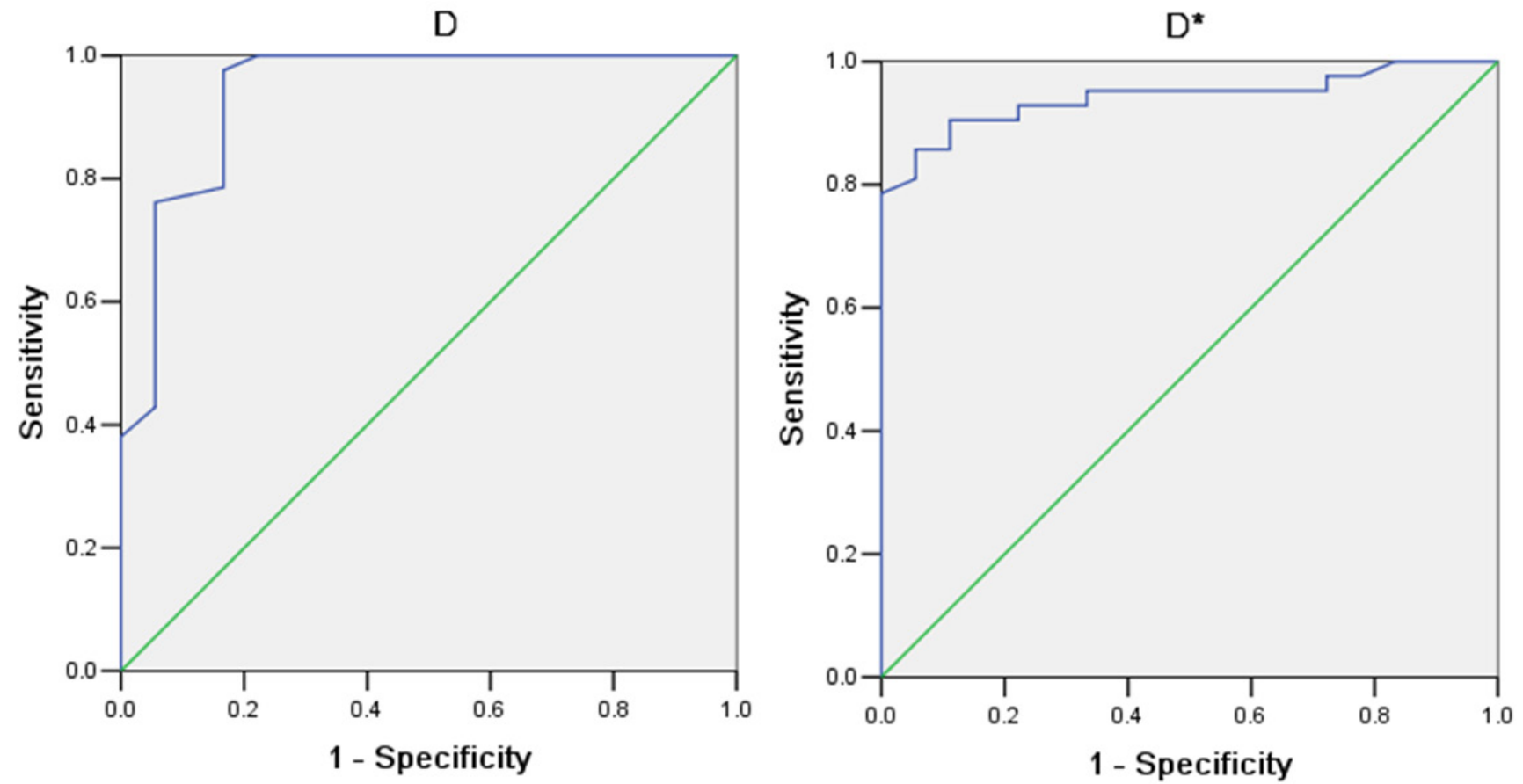

Figure 5: The ROC cure of $\boldsymbol{D}$ and $\boldsymbol{D} *$ of the two groups. Our data shows when both sensitivity and specificity were adjusted to produce the highest accuracy, the optimal $D$ and $D^{*}$ threshold for distinguishing radiosensitive NPC were $0.593 \times 10^{-3} \mathrm{~mm}^{2} / \mathrm{s}$ and $147.5 \times 10$ $3 \mathrm{~mm}^{2} / \mathrm{s}$ respectively. Besides the AUC for $D^{*}(0.942)$ was a little larger than $D(0.941)$. 
value illogic high. I even doubted that was the reason why the difference of $A D C$ value between the two groups could not reach statistical significance. In future study, when we have enlarged the number of the recruited patients, the result may probably different. Besides, we prefer to obtain a more precise value of $D$ and used 800 as the largest b value versus 1000 as the largest $b$ value in other research. I think this might also be another important reason.

In IVIM theory, $D^{*}$ is related to perfusion depended on tumor microvessel attenuation. Furthermore, the value of $D^{*}$ was determined according to the signal intensity ratios of the blood capillaries. In our study, the mean
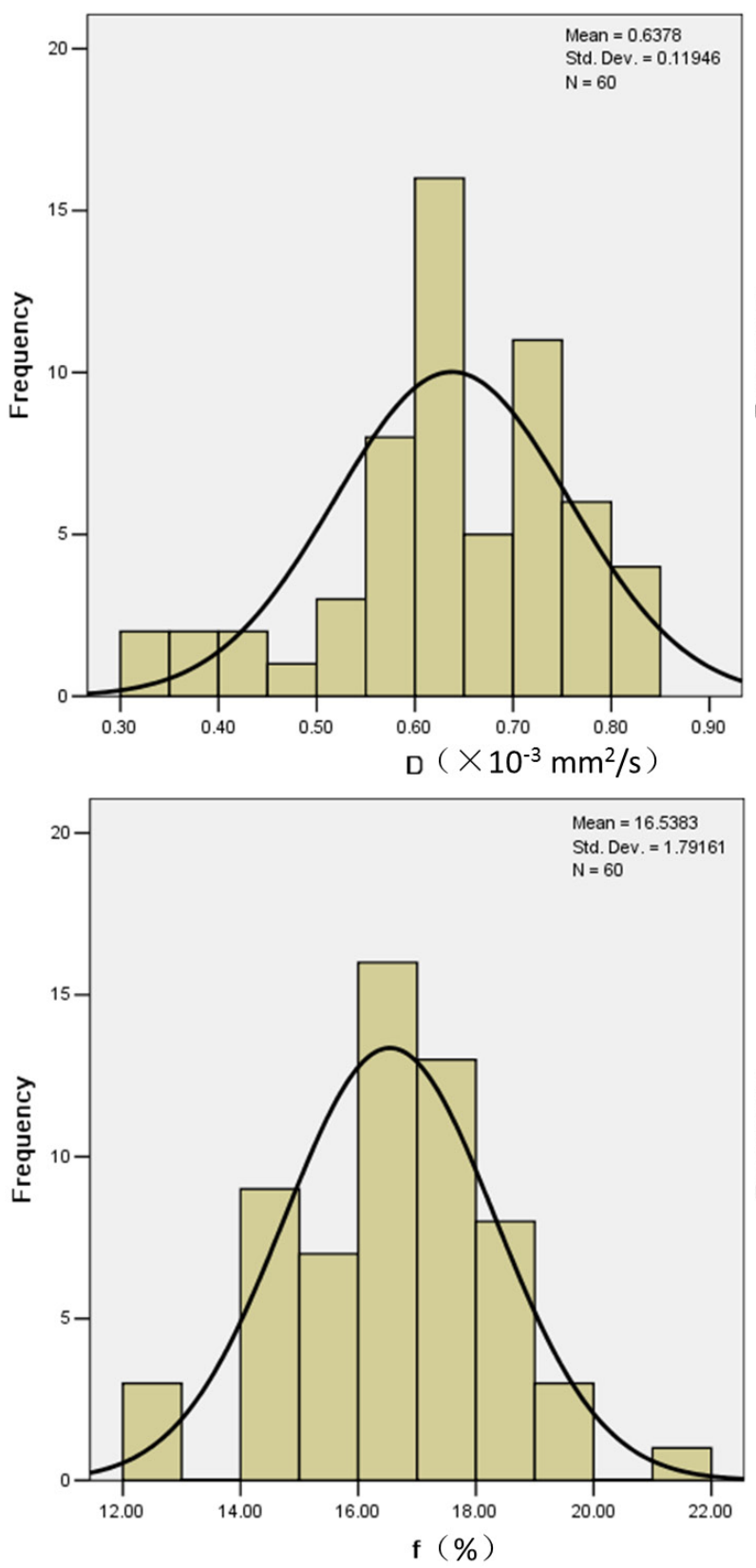

$D^{*}$ value for primary NPC was in agreement with a previous study on metastatic nodes by Lu et al.[16]. $D^{*}$ is large greater than $D$, which explains why $D^{*}$ has a stronger influence on signal decay when $b<200 \mathrm{~s} / \mathrm{mm} 2$. Our results showed a significant increase in $D^{*}$ (mean, $152.96 \times 10^{-3} \mathrm{~mm}^{2} / \mathrm{s}$ vs. $\left.120.36 \times 10^{-3} \mathrm{~mm}^{2} / \mathrm{s} ; P<0.0001\right)$ for effective group than poor effective group. This may result from the differences of histologic structure in the NPC tumor with or without abundant vessels. From the pathological slides of biopsy for the effective group, we found that there was much more neovascularization than the poor effective group. The neovessels could transport
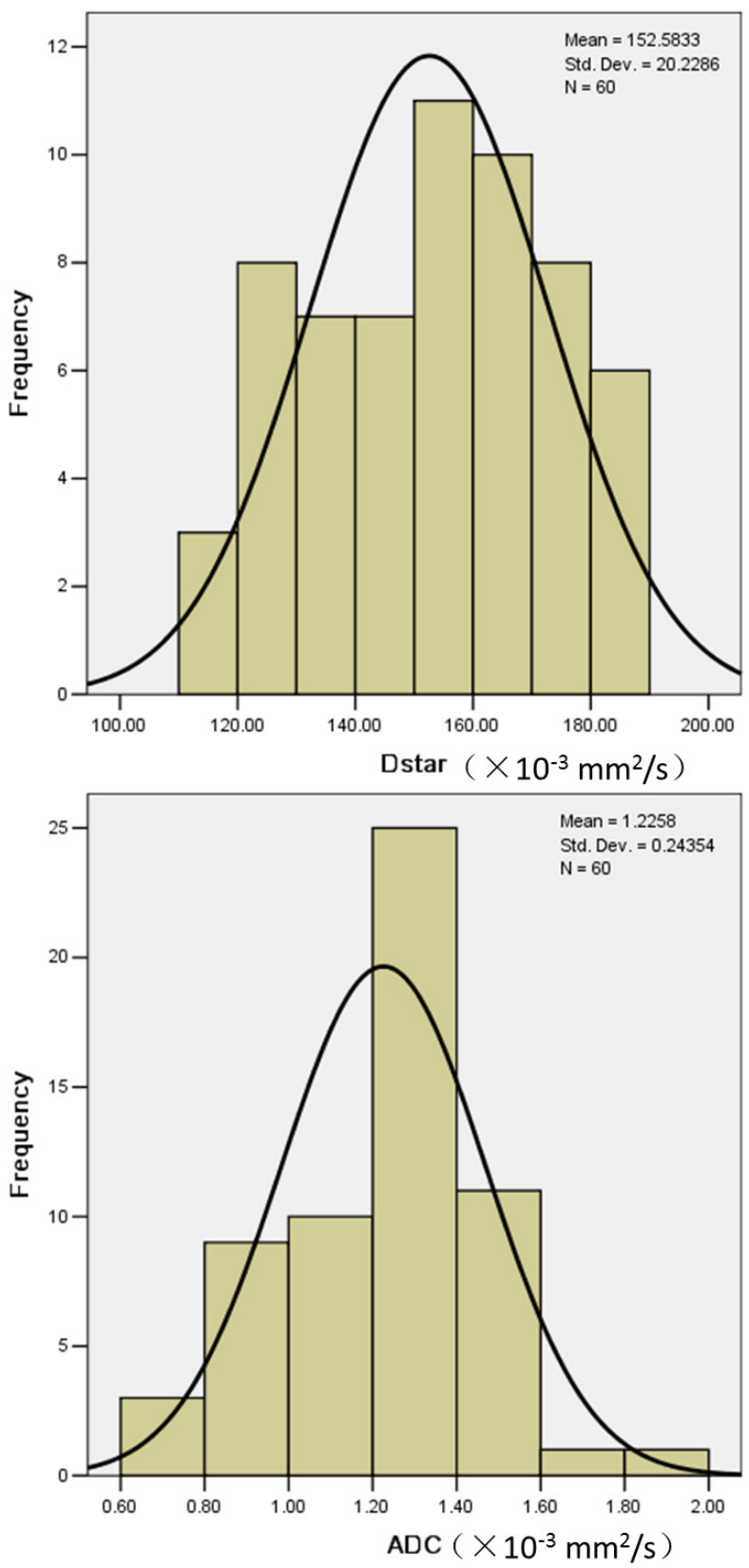

Figure 6: Histogram analysis was performed to to display the values of all the parameters and their distribution. From this histogram, it is much in evidence that all the values of $D, D^{*}$ and $f$ were relatively stable and acceptable, except $A D C$ values. The $A D C$ values of 5/18 patients in poor effective group were quirky high which made the mean ADC value illogic high. 
the blood and oxygen to the tumor lesions and improve the hypoxia. Obviously, the value of $D^{*}$ in effective groups was higher than poor effective groups, which might result from the more neovascularization in effective groups. Interestingly, the degree of capillary could be revealed by the degree of MRI enhancement in patients with NPC [34]. $D^{*}$ was considered to be proportional to the mean capillary segment length and average blood velocity [9]. So, slow blood velocity and small capillary segment length corresponded to low $D^{*}$ values. While the inadequate blood feed due to the slow blood velocity and small capillary segment length would cause the hypoxia of tumor microenvironment and poor radiosensitivity. Therefore, the increased $D^{*}$ value was proportional to the higher effective radiosensitivity. And $D^{*}$ may be an indicator for evaluating the radiosensitivity of NPC.

$\mathrm{Lu}$, et al claimed that capillary perfusion was increased in malignant tumors [35], while we found $f(18.79 \% \pm 2.51$ vs. $16.47 \% \pm 1.51 ; P>0.05)$ was not significantly different between the two groups. The similar consequence of $f$ was previously reported in the fatty liver and hepatocellular carcinoma $[25,36]$. One most convinced explanation for this consequence was from Lemke et al. [37], who demonstrated $f$ was depended on echo time. The longer echo times caused further signal decays at low b-values, and $f$ value increased. This effect would likely be significant for organs with short T2 times. However, in the IVIM sequence, relaxation effects are neglected when these relaxation times diverge, the extracted perfusion-related parameters may depend on TE and TR. Unfortunately, we did not perform T2 calculation, which made us fail to determine the "true" $f$ factor.

In order to validate our findings, we had performed the Leave-one-out tests. The classification results obtained from Leave-one out tests of original grouped cases and cross-validated grouped cases were $95.0 \%$ and $93.3 \%$. With such high correct classification, IVIM might be a reasonable model to evaluate the radiosensitivity of NPC.

Our study had certain limitations. First, a few cases of NPC, especially for the poor effective group, were recruited in this research. Second, some IVIM images contained some artifacts resulting from physiological motion and inhomogeneous magnetic field at the airbone and air-soft tissue interfaces in the skull base. Susceptibility artifact was increased with the use of higher b-values, typically approximately $1000 \mathrm{sec} / \mathrm{mm}^{2}$. we tried to minimize this effect by setting the maximum b-value as $800 \mathrm{sec} / \mathrm{mm}^{2}$. Furthermore, the poor Signal to Noise Ratio (SNR) at higher b-values may decrease the accuracy of IVIM calculation.

In conclusion, $D$ and $D^{*}$ were significantly higher in the radiosensitive NPC compared with poor radiosensitive NPC, possibly due to the restriction of molecular diffusion in poor radiosensitive NPC and increased cellularity in radiosensitive NPC. Furthermore, the significantly increased of $D^{*}$ was likely reflecting the increased blood vessel generation and parenchymal perfusion in NPC. These results demonstrated that the biexponential models of IVIM might provide a reasonable model of MRI signal decay in helping evaluate the radiosensitivity of NPC.

\section{MATERIALS AND METHODS}

\section{Patient selection treatment procedure and assessment}

This single-center study was approved by Research Ethics Committee of Guangdong Academy of Medical Sciences. Written informed consent from all patients or their guardians was obtained. From December 2011 to November 2015, 60 consecutive NPC patients (20 female, range, 27-83 years, mean age, 52 years) newly diagnosed by ENT doctor with pharyngeal mirror or nasopharyngo-fiberscope underwent general MRI and IVIM for the primary site to confirm in stage of T3 or T4. All patients accepted a standard regimen of IMRT. The prescribed dose was 69 Gy to planning target volume (PTV) of gross disease in nasopharynx and 67.5 Gy to PTV of positive lymph nodes in 30 fractions, low risk and high risk region PTV was 54 and 60 Gy in 30 fractions, respectively. All the patients were treated with one fraction daily over 5 days/week. Based on the size of locoregional lesion measured on MRI or pharyngorhinoscopy 4 weeks after IMRT, the patients were divided into the effective group and poor effective group. According to the RECIST guideline, complete response (CR) and partial response (PR) were divided into effective group, while no change (NC) and progressive disease (PD) were divided into poor group. In other words, patients with lesion complete nonresidual or lesion decreased more than $50 \%$ in area were divided into effective group. And patients with lesion decreased less than $50 \%$ or increased were divided into poor group. This study population comprised 42 patients in effective group with tumor remission (CR 35/42, PR $7 / 42$ ) and 18 patients in poor effective group with tumor enlarged or remain (SD 12/18, PD 6/18).

\section{Conventional MRI sequence}

All the patients were performed with general MRI and IVIM 1-2 days before received IMRT and 4 weeks after the end of IMRT. MRI examinations for nasopharynx were performed with a 3.0-T whole-body system (Signa EXCITE HD, GE Healthcare, Milwaukee, WI, USA) using a $40 \mathrm{mT} / \mathrm{m}$ maximum gradient capability and a standard receive-only head and neck coil. The general sequence including axial $\mathrm{T}_{1} \mathrm{WI}$ (TR/TE 600/23 $\mathrm{ms}$; $4 \mathrm{~mm}$ thickness, $1 \mathrm{~mm}$ gap; $\mathrm{NEX}=2$ ), axial and coronal contrast-enhanced $\mathrm{T}_{1} \mathrm{WI}$ after a bolus injection of gadolinium diethylenetriamine pentaacetic acid $(0.1$ 
mmol/kg, Gd-DTPA; Bayer Healthcare, Berlin, Germany), and axial $\mathrm{T}_{2}$ WI with fat suppression (TR/TE 5200/137 ms; $4 \mathrm{~mm}$ thickness, $1 \mathrm{~mm}$ gap; $\mathrm{NEX}=2$ ) using a $512 \times 288$ imaging matrix.

\section{IVIM imaging sequence}

The IVIM sequence was performed before administration of Gd-DTPA. 13 b-values $(0,10,20,30$, $50,80,100,150,200,300,400,600$ and $\left.800 \mathrm{~s} / \mathrm{mm}^{2}\right)$ were applied in the sequence with single-shot diffusionweighted spin-echo echo-planar. The lookup table of gradient directions was modified to allow multiple b-value measurements in one series. Parallel imaging was used with an acceleration factor of 2 . A local shim box covering the nasopharynx region was applied to minimize susceptibility artifacts. Totally, 14 axial slices covering the nasopharynx were obtained with a $24 \mathrm{~cm}$ field of view, $4 \mathrm{~mm}$ slice thickness, $1 \mathrm{~mm}$ slice gap, TR/ TE 3000/58 ms, matrix $128 \times 128$ and $\mathrm{NEX}=2$. The total scan time was about 225 sec.

\section{IVIM image analysis}

In the bi-exponential model of IVIM sequence, the signal intensities and $\mathrm{b}$-values are related as follows:

$\mathrm{S}_{\mathrm{b}} / \mathrm{S}_{0}=(1-f) \cdot \exp (-\mathrm{b} \cdot D)+f \cdot \exp \left(-\mathrm{b} \cdot D^{*}\right)(1)$

where $\mathrm{S}_{0}$ is the signal intensity with $b$-value of $0 ; \mathrm{S}_{\mathrm{b}}$ is the signal intensity with the b-value denoted by the subscript; $D$ is the true diffusion coefficient of a water molecule; $D^{*}$ is the pseudo-diffusion coefficient of microcirculation; and $f$ is the micro-vascular volume fraction, indicating the fraction of diffusion related to microcirculation. Based on the assumption that $D^{*}$ is roughly one order of magnitude greater than $D$ [38], its influence on signal decay can be neglected for $b>200$ $\mathrm{s} / \mathrm{mm}^{2} . f$ and $D^{*}$ were calculated by using a non-linear regression algorithm for all $b$ values. At a high $b$-value $\left(>200 \mathrm{~s} / \mathrm{mm}^{2}\right)-\mathrm{b} \cdot D^{*}$ would be less than -3 , and $f \cdot \exp (-$ $\left.\mathrm{b} \cdot D^{*}\right)$ would be less than $0.05 \cdot f$, and can therefore be neglected. In this case, Eq. (1) can be simplified as follows:

$$
\mathrm{S}_{\mathrm{b}} / \mathrm{S}_{0}=(1-f) \cdot \exp (-\mathrm{b} \cdot D)
$$

Hence, for high b-values $(300,400,600$, and $800 \mathrm{~s} /$ $\left.\mathrm{mm}^{2}\right) \mathrm{S}_{\mathrm{b}}$ was first fitted to Eq. (2) and $D$ was calculated. Although we had calculated the $f$ value previously, its accuracy was not acceptable; $f$ was recalculated using Eq. (1). Then, we fitted $S_{b}$ for all b-values using Eq. (1) with a fixed $D$ value using the nonlinear Levenberg-Marquardt method. In the fitting of Eq. (1), the initial estimated values for $f$ and $D^{*}$ were set as the previously calculated $f$ value from Eq. (2) and $10 \times 10^{-3} \mathrm{~mm}^{2} / \mathrm{s}$, respectively. Subsequently, $f$ and $D^{*}$ were obtained.

ADC value was calculated using a monoexponential fit of (SI) for all the $13 \mathrm{~b}$ values:

$$
\mathrm{S}(\mathrm{b})=\mathrm{S} 0 \exp (-\mathrm{bADC})(3)
$$

\section{Histogram analysis}

Histogram analysis was performed to display the values of all the parameters and their distribution. And such a histogram could be used to analyze the parameters voxel by voxel, thereby providing more precise information than the mean values of the parameters. Following manual lesion segmentation, histograms were generated from each parameter.Voxels for which fits generated unphysical values $(<0)$ were nulled (set to zero) for outlier rejection. Maximum and minimum values were extracted from the distribution.

\section{Statistical analysis}

The mean values of the IVIM parameters were measured independently by two experienced radiologists. They were blinded to the results of IMRT for all patients. First, the axial image section showing the primary tumor at its widest cross-section was determined using T2-weighted images as references. A largest region of interest (ROI) was then manually drawn on axial T2weighted images by each observer for each tumor at its widest section to cover as much lesions as possible while avoiding the areas of necrosis, air, large vessels, and adjacent anatomical structures (i.e., fat, muscle, and bone), and then subsequently co-registered to IVIM DWI images for further analysis. Each metric value was acquired by each observer and 2 initial data points were generated. The eventual metric value for each tumor was the mean value of the 2 initial data points.

A nonparametric Mann-Whitney test was used to assess IVIM parameters between the effective group and the poor effective group. Receiver Operating Characteristic (ROC) curves was used to estimate the diagnostic tolerance. Youden index (sensitivity-(1specificity)) was used to found the cutoff point. And we chose the $D$ and $D^{*}$ value corresponding to the max value of Youden index as the cutoff point. Intra-class correlation coefficient (ICC) analyse was performed to derive the data variability between the 2 different radiologists. Leaveone-out classification was used to validate our findings. All analyses were performed using SPSS version 13.0 for Windows (SPSS, Chicago, IL, USA). $P<0.05$ was considered statistically significant.

\section{Abbreviations}

IVIM, Intravoxel Incoherent Motion; NPC, Nasopharyngeal Carcinoma; F =Perfusion Fraction; D*= Perfusion-related Diffusion; D, Pure Molecular Diffusion; ADC, Apparent Diffusion Coefficient; ROI, Regions 
of Interest; ICC, Intraclass Correlation Coefficient; IMRT, Intensity-modulated Radiotherapy; DM, Distant Metastasis; DWI, Diffusion-weighted MR Imaging; PTV, Planning Target Volume; ROC, Receiver-operating Characteristic; SNR, Signal to Noise Ratio.

\section{Author contributions}

Wen bo Chen, Shui xing Zhang and Guan hui Cai conception and design of research; Wen bo Chen, Long Liang, and Guan hui Cai performed experiments; Wen bo Chen and Long Liang analyzed data and interpreted results of experiments; Wen bo Chen and Bin Zhang prepared figures; Wen bo Chen, Chang hong Liang and Long Liang drafted manuscript; Shui xing Zhang and Bo wen Lan edited and revised manuscript.

\section{ACKNOWLEDGMENTS}

The scientific guarantor of this publication is Shui xing. Zhang, Ph.D. The authors of this manuscript declare no relationships with any companies whose products or services may be related to the subject matter of the article. Institutional review board approval and written informed consent were obtained.

\section{CONFLICTS OF INTEREST}

ALL of the authors had no writing assistance.

\section{FUNDING}

This work was supported by the National Scientific Foundation of China (81571664), the Science and Technology Planning Project of Guangdong Province, China (2014A020212244, 2016A020216020), the Commission on Innovation and Technology of Guangdong Province, China (201605110912158), and the Clinical Research Fund of Guangdong General Hospital (2015zh04).

\section{REFERENCES}

1. Parkin DM, Whelan SL, Ferlay J, Teppo L, Thomas DB, Editors. Cancer Incidence in Five Continents, Volume III. Lyon, France: IARC Press; 2002. pp 781.

2. Jiang F, Jin T, Feng XL, Jin QF, Chen XZ. Longterm outcomes and failure patterns of patients with nasopharyngeal carcinoma staged by magnetic resonance imaging in intensity-modulated radiotherapy era: The Zhejiang Cancer Hospital's experience. J Cancer Res Ther. 2015; 11:C179-84.

3. Nordsmark M, Bentzen SM, Rudat V, Brizel D, Lartigau E, Stadler P, Becker A, Adam M, Molls M, Dunst J, Terris
DJ Overgaard J. Prognostic value of tumor oxygenation in 397 head and neck tumors after primary radiation therapy. An international multi-center study. Radiother Oncol. 2005; $77: 18-24$.

4. Vaupel P, Mayer A. Hypoxia in cancer: significance and impact on clinical outcome. Cancer Metastasis Rev. 2007; 26:225-39.

5. Jain RK. Antiangiogenesis strategies revisited: from starving tumors to alleviating hypoxia. Cancer Cell. 2014; 26:605-22.

6. Sumi M, Sakihama N, Sumi T, Morikawa M, Uetani M, Kabasawa H, Shigeno K, Hayashi K, Takahashi H, Nakamura T. Discrimination of metastatic cervical lymph nodes with diffusion-weighted MR imaging in patients with head and neck cancer. AJNR Am J Neuroradiol. 2003; 24:1627-34.

7. Razek AA, Megahed AS, Denewer A, Motamed A, Tawfik A, Nada N. Role of diffusion-weighted magnetic resonance imaging in differentiation between the viable and necrotic parts of head and neck tumors. Acta Radiol. 2008; 49:36470.

8. King AD, Ahuja AT, Yeung DK, Fong DK, Lee YY, Lei KI, Tse GM. Malignant cervical lymphadenopathy: diagnostic accuracy of diffusion-weighted MR imaging. Radiology. 2007; 245:806-13.

9. Le Bihan D, Breton E, Lallemand D, Aubin ML, Vignaud J, Laval-Jeantet M. Separation of diffusion and perfusion in intravoxel incoherent motion MR imaging. Radiology. 1988; 168:497-505.

10. Luciani A, Vignaud A, Cavet M, Nhieu JT, Mallat A, Ruel L, Laurent A, Deux JF, Brugieres P, Rahmouni A. Liver cirrhosis: intravoxel incoherent motion MR imaging — pilot study. Radiology. 2008; 249:891-99.

11. Patel J, Sigmund EE, Rusinek H, Oei M, Babb JS, Taouli B. Diagnosis of cirrhosis with intravoxel incoherent motion diffusion MRI and dynamic contrast-enhanced MRI alone and in combination: preliminary experience. J Magn Reson Imaging. 2010; 31:589-600.

12. Tshering Vogel DW, Zbaeren $\mathrm{P}$, Geretschlaeger A, Vermathen P, De Keyzer F, Thoeny HC. Diffusionweighted MR imaging including bi-exponential fitting for the detection of recurrent or residual tumour after (chemo) radiotherapy for laryngeal and hypopharyngeal cancers. Eur Radiol. 2013; 23:562-69.

13. Sumi M, Van Cauteren M, Sumi T, Obara M, Ichikawa Y, Nakamura T. Salivary gland tumors: use of intravoxel incoherent motion MR imaging for assessment of diffusion and perfusion for the differentiation of benign from malignant tumors. Radiology. 2012; 263:770-77.

14. Sumi M, Nakamura T. Head and neck tumors: assessment of perfusion-related parameters and diffusion coefficients based on the intravoxel incoherent motion model. AJNR Am J Neuroradiol. 2013; 34:410-16.

15. Guiu B, Cercueil JP. Liver diffusion-weighted MR imaging: 
the tower of Babel? Eur Radiol. 2011; 21:463-67.

16. Lu Y, Jansen JF, Mazaheri Y, Stambuk HE, Koutcher JA, Shukla-Dave A. Extension of the intravoxel incoherent motion model to non-gaussian diffusion in head and neck cancer. J Magn Reson Imaging. 2012; 36:1088-96.

17. Zhang SX, Jia QJ, Zhang ZP, Liang CH, Chen WB, Qiu $\mathrm{QH}, \mathrm{Li} \mathrm{H}$. Intravoxel incoherent motion MRI: emerging applications for nasopharyngeal carcinoma at the primary site. Eur Radiol. 2014; 24:1998-2004.

18. Cho GY, Moy L, Kim SG, Baete SH, Moccaldi M, Babb JS, Sodickson DK, Sigmund EE. Evaluation of breast cancer using intravoxel incoherent motion (IVIM) histogram analysis: comparison with malignant status, histological subtype, and molecular prognostic factors. Eur Radiol. 2015.

19. Bisdas S, Klose U. IVIM analysis of brain tumors: an investigation of the relaxation effects of CSF, blood, and tumor tissue on the estimated perfusion fraction. MAGMA. $2015 ; 28: 377-83$.

20. Cui Y, Dyvorne H, Besa C, Cooper N, Taouli B. IVIM Diffusion-weighted Imaging of the Liver at 3.0T: comparison with 1.5T. Eur J Radiol Open. 2015; 2:123-28.

21. Moore RJ, Issa B, Tokarczuk P, Duncan KR, Boulby P, Baker PN, Bowtell RW, Worthington BS, Johnson IR, Gowland PA. In vivo intravoxel incoherent motion measurements in the human placenta using echo-planar imaging at 0.5 T. Magn Reson Med. 2000; 43:295-302.

22. Qi J, Olsen NJ, Price RR, Winston JA, Park JH. Diffusion-weighted imaging of inflammatory myopathies: polymyositis and dermatomyositis. J Magn Reson Imaging. 2008; 27:212-17.

23. Riches SF, Hawtin K, Charles-Edwards EM, de Souza NM. Diffusion-weighted imaging of the prostate and rectal wall: comparison of biexponential and monoexponential modelled diffusion and associated perfusion coefficients. NMR Biomed. 2009; 22:318-25.

24. Liu C, Liang C, Liu Z, Zhang S, Huang B. Intravoxel incoherent motion (IVIM) in evaluation of breast lesions: comparison with conventional DWI. Eur J Radiol. 2013; 82:e782-89.

25. Guiu B, Petit JM, Capitan V, Aho S, Masson D, Lefevre $\mathrm{PH}$, Favelier S, Loffroy R, Vergès B, Hillon P, Krausé $\mathrm{D}$, Cercueil JP. Intravoxel incoherent motion diffusionweighted imaging in nonalcoholic fatty liver disease: a 3.0T MR study. Radiology. 2012; 265:96-103.

26. Loncaster JA, Carrington BM, Sykes JR, Jones AP, Todd SM, Cooper R, Buckley DL, Davidson SE, Logue JP, Hunter RD, West CM. Prediction of radiotherapy outcome using dynamic contrast enhanced MRI of carcinoma of the cervix. Int J Radiat Oncol Biol Phys. 2002; 54:759-67.

27. Hawighorst H, Knapstein PG, Knopp MV, Weikel W, Brix G, Zuna I, Schönberg SO, Essig M, Vaupel P, van Kaick G. Uterine cervical carcinoma: comparison of standard and pharmacokinetic analysis of time-intensity curves for assessment of tumor angiogenesis and patient survival. Cancer Res. 1998; 58:3598-602.

28. Thoeny HC, De Keyzer F, King AD. Diffusion-weighted MR imaging in the head and neck. Radiology. 2012; 263:19-32.

29. Kim S, Loevner L, Quon H, Sherman E, Weinstein G, Kilger A Poptani H. Diffusion-weighted magnetic resonance imaging for predicting and detecting early response to chemoradiation therapy of squamous cell carcinomas of the head and neck. Clin Cancer Res. 2009; 15:986-994. https:// doi.org/10.1158/1078-0432.CCR-08-1287.

30. Hompland T, Ellingsen C, Galappathi K, Rofstad EK. DW-MRI in assessment of the hypoxic fraction, interstitial fluid pressure, and metastatic propensity of melanoma xenografts. BMC Cancer. 2014; 14:92.

31. Padhani AR. Diffusion magnetic resonance imaging in cancer patient management. Semin Radiat Oncol. 2011; 21:119-40.

32. Yankeelov TE, Arlinghaus LR, Li X, Gore JC. The role of magnetic resonance imaging biomarkers in clinical trials of treatment response in cancer. Semin Oncol. 2011; 38:1625.

33. Bonekamp S, Corona-Villalobos CP, Kamel IR. Oncologic applications of diffusion-weighted MRI in the body. J Magn Reson Imaging. 2012; 35:257-79.

34. White ML, Zhang Y, Robinson RA. Evaluating tumors and tumorlike lesions of the nasal cavity, the paranasal sinuses, and the adjacent skull base with diffusion-weighted MRI. J Comput Assist Tomogr. 2006; 30:490-95.

35. Lu CH, Yang CY, Wang CP, Yang CC, Liu HM, Chen YF. Imaging of nasopharyngeal inflammatory pseudotumours: differential from nasopharyngeal carcinoma. Br J Radiol. 2010; 83:8-16.

36. Lewin M, Fartoux L, Vignaud A, Arrivé L, Menu Y, Rosmorduc $\mathrm{O}$. The diffusion-weighted imaging perfusion fraction $\mathrm{f}$ is a potential marker of sorafenib treatment in advanced hepatocellular carcinoma: a pilot study. Eur Radiol. 2011; 21:281-90.

37. Lemke A, Laun FB, Simon D, Stieltjes B, Schad LR. An in vivo verification of the intravoxel incoherent motion effect in diffusion-weighted imaging of the abdomen. Magn Reson Med. 2010; 64:1580-85.

38. Le Bihan D. Intravoxel incoherent motion perfusion MR imaging: a wake-up call. Radiology. 2008; 249:748-52. 